02

\title{
Расчет ядерных тормозных способностей в квазиклассическом приближении
}

\author{
(С) П.Ю. Бабенко, А.Н. Зиновьев
}

Физико-технический институт им. А.Ф. Иофрфе РАН, 94021 Санкт-Петербург, Россия

e-mail: babenko@npd.ioffe.ru

Поступило в Редакцию 29 июня 2021 г.

В окончательной редакции 24 июля 2021 г.

Принято к публикации 27 июля 2021 г.

Представлены результаты расчета ядерных тормозных способностей в квазиклассическом приближении для систем $\mathrm{H}-\mathrm{Be}, \mathrm{H}-\mathrm{C}, \mathrm{H}-\mathrm{W}, \mathrm{O}-\mathrm{C}, \mathrm{O}-\mathrm{Be}, \mathrm{O}-\mathrm{Al}$. Обнаружено, что при наличии ямы в потенциале межатомного взаимодействия появляется дополнительный максимум на зависимости ядерных тормозных способностей от энергии бомбардирующих частиц. При использовании универсального потенциала без ямы эта особенность отсутствует. Показано, что масштабированием полученные данные для систем с водородом пересчитываются для столкновений с участием изотопов водорода D и Т. Полученные результаты хорошо согласуются с классическими расчетами, что объясняется тем фактом, что в ядерные тормозные способности вносят основной вклад большие углы рассеяния, и критерии применимости меняются на условие „угловой момент $l \gg 1^{\prime \prime}$.

Ключевые слова: ядерные тормозные способности, квазиклассическое приближение, потенциалы межатомного взаимодействия.

DOI: $10.21883 / J T F .2021 .12 .51754 .199-21$

\section{Введение}

Знание потерь энергии при торможении частиц в веществе необходимо для ионной имплантации, при производстве тонких пленок, для развития методов анализа поверхности, при расчетах воздействия плазмы на стенки термоядерных устройств, при повреждении материалов в ядерных реакторах. Для описания торможения частиц в веществе используется подход, который различает потери энергии, связанные с упругим рассеянием налетающей частицы на ядрах атомов мишени (ядерные тормозные способности, (ЯТС)), и электронные тормозные способности (ЭТС), связанные с возбуждением и ионизацией взаимодействующих атомов. Для легких атомов при энергиях выше нескольких $\mathrm{keV}$ доминируют ЭТС. В пристеночной плазме токамака, где энергии частиц составляют $10-200 \mathrm{eV}$, доминируют ЯТС. Понимание взаимодействия частиц плазмы с первой стенкой токамака является одной из основных проблем, которые необходимо решить для успешной работы токамакареактора. В проекте ИТЭР планируется использование дивертора из $\mathrm{W}$, основная камера будет покрыта слоем Ве, в ряде токамаков используется углерод, что объясняет выбор систем $\mathrm{H}-\mathrm{W}, \mathrm{H}-\mathrm{Be}$ и $\mathrm{H}-\mathrm{C}$. Также приведены расчеты для ряда систем с кислородом $\mathrm{O}-\mathrm{Be}, \mathrm{O}-\mathrm{C}, \mathrm{O}-\mathrm{Al}$.

К сожалению, авторам не удалось найти в литературе данных по экспериментальному определению ЯТС. В работах [1-3] измерялись полные потери энергии частиц, при этом для учета вклада ЯТС использовались расчеты с помощью кода SRIM, которые приводят к заметным погрешностям. Анализ влияния различных методов расчета ЯТС на результаты экспериментов по определению пробегов частиц в веществе и процессы ионной имплантации проводился в работах $[4,5]$.

Расчеты ЯТС методами классической механики для систем $\mathrm{H}-\mathrm{Be}, \mathrm{H}-\mathrm{C}, \mathrm{H}-\mathrm{W}$ для области энергий соударения $E>10 \mathrm{eV}$ были выполнены в нашей работе [6]. При меньших энергиях длина волны Де-Бройля для протонов становится сравнимой с размерами атомов, поэтому для уточнения расчетов и расширения диапазона энергий до $0.1 \mathrm{eV}$ были выполнены расчеты с использованием квазиклассического приближения.

\section{1. Применимость квазиклассического приближения}

Применимость квазиклассического рассмотрения задачи сводится к условию

$$
\frac{1}{2 \pi}\left|\frac{d \lambda}{d x}\right| \ll 1,
$$

где $\lambda(x)=2 \pi \hbar / p(x)$ - дебройлевская длина волны частицы, $x-$ координата частицы. Таким образом, длина волны частицы должна мало меняться на протяжении расстояний, характерных для изменения потенциала. В нашем случае это расстояние совпадает с размером потенциальной ямы $a \sim 1 \AA$. Критерий $\lambda / 2 \pi<0.1 a$ выполняется для атома водорода вплоть до энергий $E>0.3 \mathrm{eV}$. 
Если вернуться к критерию применимости классических расчетов для вычисления транспортного сечения или ядерных тормозных потерь, то обычный критерий применимости „классики“ для описания рассеяния $\theta l \gg 1$, где $l-$ угловой момент, в случае расчета транспортного сечения или ЯТС сводится к более простому условию $l \gg 1$, так как основной вклад в сечение ЯТС вносят углы $\theta \sim 1$.

\section{2. Методика расчета}

Сечение ядерного торможения выражается формулой [7]:

$$
S_{n}=\frac{d E}{d R} \frac{1}{N}=\int_{0}^{\infty} 2 \pi b T[\theta(b)] d b
$$

где $N$ - плотность мишени. Потеря энергии при соударении $T[\theta]$ дается формулой

$$
T[\theta(b)]=\frac{4 M_{1} M_{2}}{\left(M_{1}+M_{2}\right)^{2}} E \sin ^{2}\left[\frac{\theta(b)}{2}\right] .
$$

Здесь $M_{1}$ и $M_{2}$ - массы сталкивающихся частиц, $\theta(b)-$ угол рассеяния в системе центра масс (СЦМ) при параметре удара $b, E-$ энергия налетающей частицы.

Таким образом:

$$
S_{n}=4 \frac{M_{1} M_{2}}{\left(M_{1}+M_{2}\right)^{2}} E \int_{0}^{\infty} 2 \pi \sin ^{2}\left[\frac{\theta(b)}{2}\right] b d b .
$$

Транспортное сечение связано с сечением рассеяния на угол $\theta$ соотношением [8]:

$$
Q_{t r}=\int_{0}^{\infty}(1-\cos \theta) d \sigma=2 \int_{0}^{\infty} 2 \pi \sin ^{2}\left[\frac{\theta(b)}{2}\right] b d b .
$$

Отсюда сечение ядерного торможения $S_{n}$ выражается через транспортное сечение $Q_{t r}$ следующим образом:

$$
S_{n}=Q_{t r} \frac{2 M_{1} M_{2}}{\left(M_{1}+M_{2}\right)^{2}} E .
$$

В классической механике сечение рассеяния рассчитывается через зависимость угла рассеяния от параметра удара при использовании конкретного потенциала взаимодействия. В квазиклассическом приближении понятие траектории отсутствует.

Для расчета транспортного сечения в квазиклассическом приближении обычно применяется формула [9]:

$$
Q_{t r}=\frac{4 \pi}{k^{2}} \sum_{l=0}^{\infty}(l+1) \sin ^{2}\left(\delta_{l}-\delta_{l+1}\right) .
$$

Здесь $k=p / \hbar-$ волновой вектор, $p-$ импульс в СЦМ, $l-$ угловой момент, $\delta_{l}-$ фаза рассеяния.
Эта формула отличается от оригинальной формулы Месси и Мора, приведенной в работе [10]. Подробного вывода формулы (7) в литературе, доступной в интернете, нам найти не удалось, поэтому в Приложении приводим наш вывод широко используемой формулы.

Фаза $\delta_{l}$ в квазиклассическом приближении определяется выражением [8]:

$$
\begin{aligned}
\delta_{l}= & \int_{r_{0}}^{\infty}\left[\sqrt{2 \mu\left[E_{c m s}-U(r)\right]-\frac{\left(l+\frac{1}{2}\right)^{2}}{r^{2}}}-k\right] d r \\
& +\frac{\pi}{2}\left(l+\frac{1}{2}\right)-k r_{0},
\end{aligned}
$$

где $r$ - межъядерное расстояние, $\mu$ - приведенная мacca $\left(\mu=M_{1} M_{2} /\left\{M_{1}+M_{2}\right\}\right), E_{c m s}$ - энергия в СЦМ, $U$ - потенциал межатомного взаимодействия, $r_{0}-$ корень уравнения

$$
2 \mu\left[E_{c m s}-U\left(r_{0}\right)\right]-\frac{\left(l+\frac{1}{2}\right)^{2}}{r_{0}^{2}}=0 .
$$

Как видно, в подынтегральном выражении в формуле (8) вычитаются два больших члена. Чтобы увеличить точность вычислений, мы преобразовали выражение для фазы, умножив и разделив подынтегральное выражение на величину

$$
\sqrt{2 \mu\left[E_{c m s}-U(r)\right]-\frac{\left(l+\frac{1}{2}\right)^{2}}{r^{2}}}+k,
$$

а также, чтобы ограничить диапазон интегрирования, сделали замену переменной $t=r_{0} / r$. В результате получаем

$$
\begin{aligned}
\delta_{l}\left(r_{0}, l\right)= & \int_{0}^{1} \frac{\left[-2 \mu U\left(\frac{r_{0}}{t}\right)-\frac{\left(l+\frac{1}{2}\right)^{2}}{\left(\frac{r_{0}}{t}\right)^{2}}\right]\left(\frac{r_{0}}{t^{2}}\right)}{\sqrt{k^{2}-2 \mu U\left(\frac{r_{0}}{t}\right)-\frac{\left(l+\frac{1}{2}\right)^{2}}{\left(\frac{r_{0}}{t}\right)^{2}}+k}} d t \\
& +\frac{\pi}{2}\left(l+\frac{1}{2}\right)-k r_{0} .
\end{aligned}
$$

Максимальное число $l$ в сумме при вычислении транспортного сечения выбиралось большим, достаточным, чтобы не влиять на результаты расчета.

Как видно из приведенных формул, как при расчетах классических траекторий, так и фазы, определяющее значение имеет выбор потенциала для адекватного описания системы взаимодействующих частиц.

\section{3. Потенциалы межатомного взаимодействия}

При компьютерном моделировании взаимодействия частиц с твердым телом часто используется „универ- 


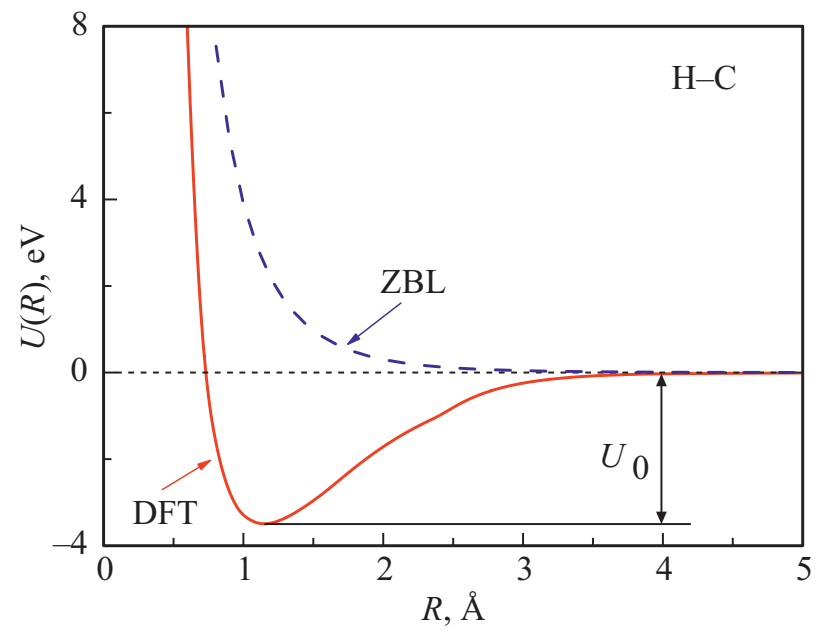

Рис. 1. Зависимость потенциала взаимодействия от расстояния между атомами для пары Н-C. Штриховая линия потенциал ZBL, сплошная линия - потенциал DFT. Глубина ямы в потенциале обозначена $U_{0}$.

сальный“ потенциал ZBL [11]. При более точных расчетах используются потенциалы, полученные в приближении функционала плотности (DFT - потенциалы, DFT - density function theory) [12]. На рис. 1. приведены потенциалы ZBL и DFT для системы $\mathrm{H}-\mathrm{C}$ в зависимости от расстояния между атомами. В потенциале ZBL яма отсутствует, а в потенциале DFT для системы $\mathrm{H}-\mathrm{C}$ присутствует яма глубиной $U_{0}=3.5 \mathrm{eV}$.

В наших расчетах мы использовали потенциалы DFT [12] с уточнением глубины потенциальной ямы по измерениям энергии диссоциации молекул [13-17].

\section{4. Результаты расчета}

Результаты расчетов ядерных тормозных потерь в квазиклассическом приближении приведены на рис. 2 . Они хорошо согласуются с расчетом по классической механике, когда применимо понятие траектории.

Совпадение результатов расчетов по классической механике и в квазиклассическом приближении связано с тем фактом, что при расчете транспортного сечения критерий применимости $l \gg 1$ выполняется вплоть до тепловых энергий.

Для всех систем с потенциальной ямой в зависимости сечения ЯТС наблюдается дополнительный пик при небольших энергиях соударения (рис. 2-6).

Видно, что при наличии ямы в потенциале взаимодействия в зависимости сечения ядерного торможения появляется максимум. То есть использование универсального потенциала ZBL может приводить к существенным ошибкам в определении тормозных способностей частиц. Наличие дополнительного максимума должно обязательно учитываться при расчете тормозных потерь частиц в пристеночной плазме токамака-реактора.
На рис. 5 представлены расчеты ЯТС для систем $\mathrm{H}-\mathrm{W}, \mathrm{D}-\mathrm{W}, \mathrm{T}-\mathrm{W}$, т.е. для систем с различными изотопами водорода.

Как показано в нашей работе [12], потенциалы взаимодействия для различных изотопов водорода практически совпадают. Это позволяет пересчитать транспортное сечение и сечение ЯТР для этих случаев простой перенормировкой, как это продемонстрировано в нашей работе [6]. На рис. 6 представлены те же сечения в приведенных координатах $\varepsilon=E / \varepsilon_{0}$ и $S=S_{e} / S_{0}$, где $\varepsilon_{0}=\left(Z_{1} Z_{2} e^{2} / a\right)\left(M_{1}+M_{2}\right) / M_{2}, \quad S_{0}=\pi a^{2} \varepsilon_{0} 4 M_{1} M_{2} /\left(M_{1}+\right.$ $\left.+M_{2}\right)^{2}$, предложенных в [18]. Выбор длины экранирования а зависит от типа потенциала. В нашем случае применяется длина экранирования Фирсова [19], выраженная в атомных единицах, $a_{\mathrm{F}}=0.8853 /\left(Z_{1}^{1 / 2}+Z_{2}^{1 / 2}\right)^{2 / 3}$, здесь $Z_{1}$ и $Z_{2}$ - заряды ядер сталкивающихся частиц.

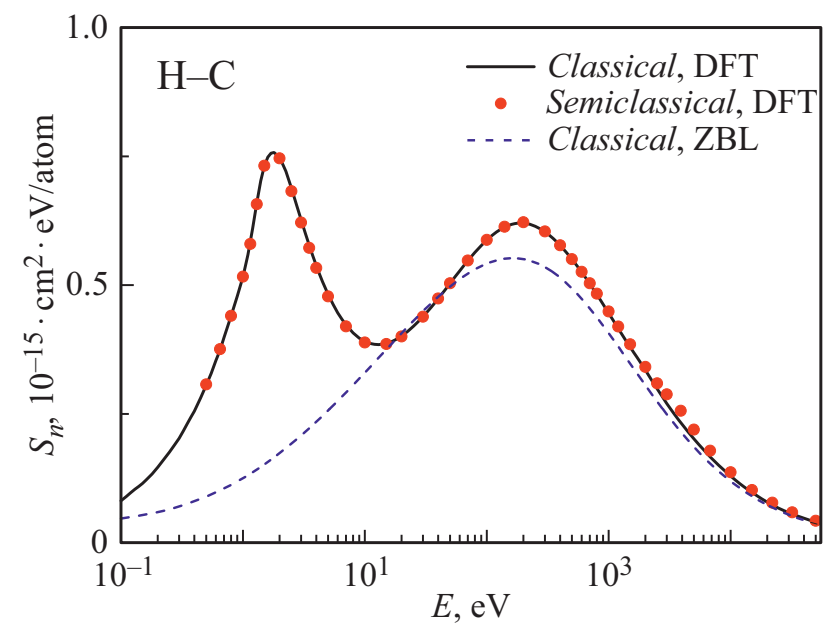

Рис. 2. Зависимость сечения ядерного торможения от энергии налетающей частицы для пары Н-C. Штриховая линия „классика“ и потенциал ZBL, сплошная линия - „классика“ и потенциал DFT, точки - „квазиклассика“ и потенциал DFT.

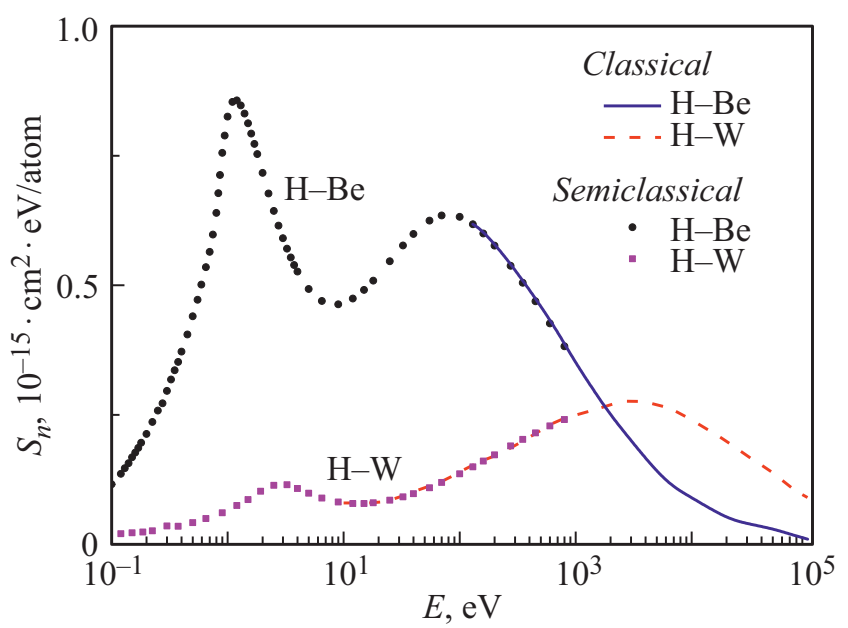

Рис. 3. Зависимость сечения ядерного торможения от энергии налетающей частицы для пары $\mathrm{H}-\mathrm{Be}$ и $\mathrm{H}-\mathrm{W}$. Линии „классика“ и потенциал DFT, точки - „Квазиклассика“ и потенциал DFT. 


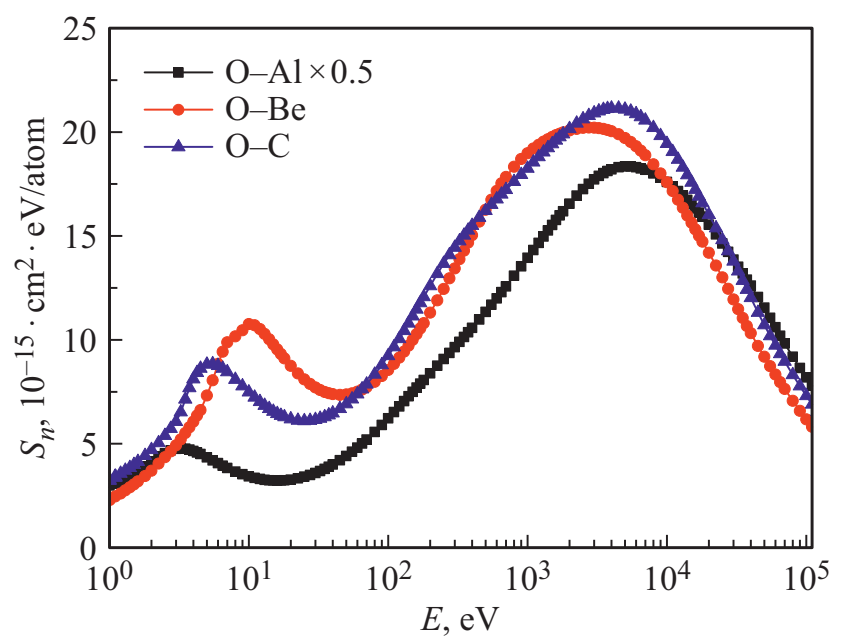

Рис. 4. Зависимость сечения ЯТС от энергии соударения для различных систем с кислородом.

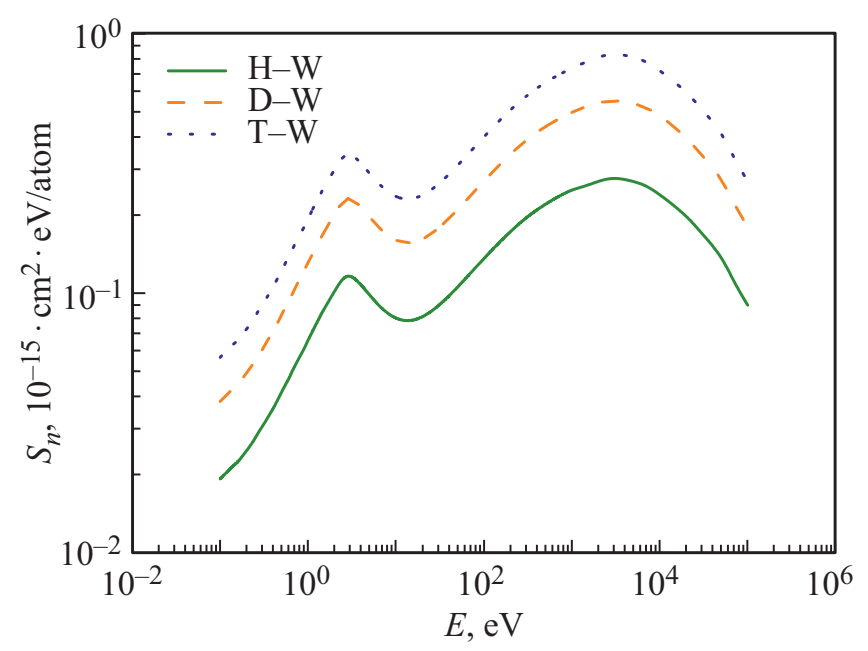

Рис. 5. ЯТС для систем $\mathrm{H}-\mathrm{W}, \mathrm{D}-\mathrm{W}, \mathrm{T}-\mathrm{W}$.

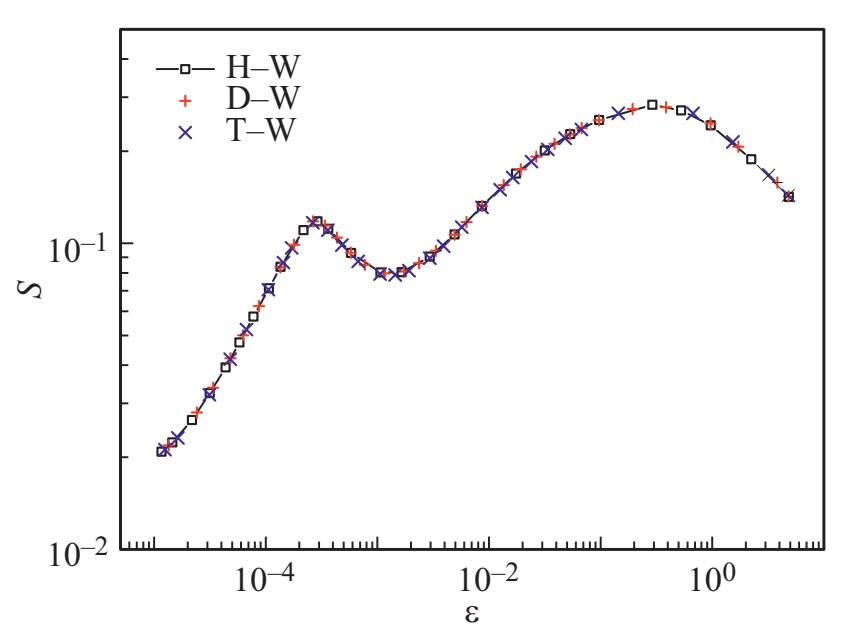

Рис. 6. Данные о ЯТС для тех же систем в универсальных координатах.
Как видно из рис. 6, данные для разных изотопов описываются универсальной кривой. Аналогично можно пересчитать данные для разных изотопов и для систем с Ве и С.

\section{Заключение}

Выполнены расчеты сечений ЯТС для систем $\mathrm{H}-\mathrm{Be}$, $\mathrm{H}-\mathrm{C}, \mathrm{H}-\mathrm{W}$, представляющие интерес для расчета процессов в пристеночной области токамака-реактора, а также для некоторых систем с кислородом $(\mathrm{O}-\mathrm{C}, \mathrm{O}-\mathrm{Be}$, $\mathrm{O}-\mathrm{Al})$. Установлено, появление дополнительного пика в зависимости сечения ЯТС от энергии соударения, связанного с рассеянием на притягивающей яме в потенциале.

Представляется важным практическое совпадение результатов, полученных в квазиклассическом приближении, и данных, полученных методами классической механики. Это позволяет значительно упростить расчеты для других систем.

Установлено, что сечения ЯТС для различных изотопов водорода пересчитываются простой перенормировкой.

Следует обратить внимание, что расчеты ЯТС в настоящей работе выполнены для рассеяния на потенциале, соответствующем основному состоянию системы. При рассмотрении столкновений частиц следует учитывать многоканальность рассеяния, т.е. возможность одновременного рассеяния на множестве термов, соответствующих как основному, так и возбужденным состояниям системы. Для энергий свыше $100 \mathrm{eV}$ это различие малосущественно, в то время как при меньших энергиях влияние притяжения слабее сказывается на потенциалах, описывающих рассеяние на возбужденных состояниях. Соответственно влияние дополнительного пика будет менее выражено. Мы планируем учесть поправки, связанные с многоканальным рассеянием в будущем. Основная трудность здесь связана с получением надежных данных о потенциалах для возбужденных состояний.

\section{Приложение. Вывод формулы для транспортного сечения $Q_{t r}$}

$$
Q_{t r}=2 \pi \int_{0}^{\pi} d \theta \sin \theta(1-\cos \theta)|f(\theta)|^{2} .
$$

Здесь $\theta-$ угол рассеяния в системе центра масс, $f(\theta)$ - амплитуда рассеяния, которая без каких-либо предположений и упрощений может быть записана 
в виде

$$
f=\frac{1}{k} \sum_{l=0}^{\infty} e^{i \delta_{l}}(2 l+1) P_{l}(\cos \theta) \sin \delta_{l},
$$

где $\delta_{l}-$ фаза рассеяния.

$$
\begin{aligned}
& Q_{t r}=2 \pi \int_{0}^{\pi} d \theta \sin \theta(1-\cos \theta) \mid \frac{1}{4 k^{2}}\left(\sum_{l=0}^{\infty} e^{i \delta_{l}}(2 l+1)\right. \\
& \left.\times P_{l}(\cos \theta) \sin \delta_{l}\right)\left(\sum_{m=0}^{\infty} e^{-i \delta_{m}}(2 m+1) P_{m}(\cos \theta) \sin \delta_{m}\right) \mid
\end{aligned}
$$

Для члена с единицей получаем слагаемое, соответствующее сечению упругого рассеяния. Для члена c $\cos \theta$ воспользуемся свойствами полиномов Лежандра:

$$
\cos \theta P_{l}(\cos \theta)=\frac{(l+1) P_{l+1}(\cos \theta)}{2 l+1}+\frac{l P_{l-1}(\cos \theta)}{2 l+1}
$$

В произведении двух сумм вследствие ортогональности полиномов Лежандра в сумме по $l$ останутся вклады от членов с $m=l-1$ и $l+1$.

Рассмотрим член с $m=l-1$ :

$(2 l-1) \cos \theta P_{l-1}(\cos \theta)=(l) P_{l}(\cos \theta)+(l-1) P_{l-2}(\cos \theta)$.

Рассмотрим член с $m=l+1$ :

$$
\begin{aligned}
& (2 l+3) \cos \theta P_{l+1}(\cos \theta)=(l+2) P_{l+2}(\cos \theta) \\
& \quad+(l+1) P_{l}(\cos \theta) .
\end{aligned}
$$

При интегрировании, в силу ортогональности полиномов Лежандра, если рассматривать член с индексом $l$ в первой сумме, то из второй суммы в случае $m=l-1$ вносит вклад только первый член в (П5), а для члена с $m=l+1$ - только второй член в (П6).

Перепишем сумму

$$
\begin{aligned}
& Q_{t r}=\frac{4 \pi}{k^{2}} \sum_{l=0}^{\infty}(2 l+1) \sin ^{2} \delta_{l}-2 \pi \frac{1}{k^{2}} \int_{0}^{\pi} d \theta \sin \theta \\
& \times \mid\left(\sum_{l=0}^{\infty} e^{i \delta_{l}}(2 l+1) P_{l}(\cos \theta) \sin \delta_{l}\right)\left((l) P_{l}(\cos \theta) \sin \delta_{l-1}\right. \\
& \left.\times e^{-i \delta_{l-1}}+(l+1) P_{l}(\cos \theta) \sin \delta_{l+1} e^{-i \delta_{l+1}}\right) \mid
\end{aligned}
$$

Учитывая нормировку полиномов Лежандра

$$
\int_{0}^{\pi} d \theta \sin \theta P_{l}(\cos \theta) P_{l}(\cos \theta)=\frac{2}{2 l+1},
$$

получаем

$$
\begin{aligned}
& Q_{t r}=\frac{4 \pi}{k^{2}} \sum_{l=0}^{\infty}(2 l+1) \sin ^{2} \delta_{l}-\frac{4 \pi}{k^{2}} \mid \sum_{l=0}^{\infty}\left(l \sin \delta_{l} \sin \delta_{l-1}\right. \\
& \left.\times e^{i\left(\delta_{l}-\delta_{l-1}\right)}+(l+1) \sin \delta_{l} \sin \delta_{l+1} e^{i\left(\delta_{l}-\delta_{l+1}\right)}\right) \mid
\end{aligned}
$$

При $l=0$ член во второй сумме вклада не дает. Сдвигая индекс суммирования, получае

$$
\begin{aligned}
Q_{t r}=\frac{4 \pi}{k^{2}} \sum_{l=0}^{\infty}(2 l+1) \sin ^{2} \delta_{l}-\frac{4 \pi}{k^{2}} \mid \sum_{l=0}^{\infty}(l+1) \sin \delta_{l+1} \sin \delta_{l} \\
\times\left(e^{i\left(\delta_{l+1}-\delta_{l}\right)}+e^{i\left(\delta_{l}-\delta_{l+1}\right)}\right) \mid .
\end{aligned}
$$

Разбив первую сумму на две с весами $l$ и $l+1$ и сдвинув суммирование в первом члене на единицу, имеем

$$
\begin{aligned}
& Q_{t r}=\frac{4 \pi}{k^{2}} \sum_{l=0}^{\infty}(l+1)\left(\sin ^{2} \delta_{l}+\sin ^{2} \delta_{l+1}\right) \\
& -\frac{4 \pi}{k^{2}}\left|\sum_{l=0}^{\infty}(l+1) \sin \delta_{l+1} \sin \delta_{l} 2 \cos \left(\delta_{l+1}-\delta_{l}\right)\right| .
\end{aligned}
$$

Объединяя суммы, получаем

$$
\begin{aligned}
Q_{t r}= & \frac{4 \pi}{k^{2}} \sum_{l=0}^{\infty}(l+1)\left\{\sin ^{2} \delta_{l}+\sin ^{2} \delta_{l+1}-2 \sin \delta_{l+1} \sin \delta_{l}\right. \\
& \left.\times\left(\cos \delta_{l+1} \cos \delta_{l}+\sin \delta_{l+1} \sin \delta_{l}\right)\right\}
\end{aligned}
$$

или

$$
\begin{aligned}
Q_{t r}= & \frac{4 \pi}{k^{2}} \sum_{l=0}^{\infty}(l+1)\left\{\sin ^{2} \delta_{l} \cos ^{2} \delta_{l+1}-2 \sin \delta_{l+1} \sin \delta_{l}\right. \\
& \left.\times \cos \delta_{l+1} \cos \delta_{l}+\sin ^{2} \delta_{l+1} \cos ^{2} \delta_{l}\right\} .
\end{aligned}
$$

Проведя тригонометрические преобразования, получим формулу Мотта-Месси [9]:

$$
Q_{t r}=\frac{4 \pi}{k^{2}} \sum_{l=0}^{\infty}(l+1) \sin ^{2}\left(\delta_{l}-\delta_{l+1}\right) .
$$

\section{Финансирование работы}

Работа выполнена в рамках государственного задания Министерства образования и науки РФ для Федерального государственного бюджетного учреждения науки Физико-технического института им. А.Ф. Иоффе РАН.

\section{Конфликт интересов}

Авторы заявляют, что у них нет конфликта интересов. 


\section{Список литературы}

[1] H. Grahmann, S. Kalbitzer. NIM, 132, 119 (1976).

DOI: $10.1016 / 0029-554 X(76) 90720-5$

[2] D. Jedrejcic, U. Greife. NIMB, 428, 1 (2018).

DOI: 10.1016/j.nimb.2018.04.039

[3] R. Shnidman, R.M. Tapphorn, K.N. Geller. Appl. Phys. Lett., 22, 551 (1973). DOI: 10.1063/1.1654504

[4] L.G. Glazov, P. Sigmund. NIMB, 207, 240 (2003). DOI: $10.1016 / \mathrm{S} 0168-583 \mathrm{X}(03) 00461-0$

[5] Th. Krist, P. Mertens, J.P. Biersack. NIMB, 2, 177 (1984). DOI: $10.1016 / 0168-583 X(84) 90183-6$

[6] А.Н. Зиновьев, П.Ю. Бабенко. Письма в ЖТФ, 46, 23 (2020). DOI: 10.21883/PJTF.2020.18.49997.18265 [A.N. Zinoviev, P.Yu. Babenko, Tech. Phys. Lett., 46, 909 (2020). DOI: 10.1134/S106378502009031X]

[7] Х. Риссел, И. Руге. Ионная имплантация (Наука, М., 1983) [H. Ryssel, I. Ruge. Ionenimplantation (Vieweg and Teubner Verlag, Stuttgart, 1978)]

[8] Л.Д. Ландау, Е.М. Лифшиц. Квантовая механика (Наука, M., 1989) [L.D. Landau, E.M. Lifshitz. Quantum Mechanics (Pergamon Press, London, 1965)]

[9] Н. Мотт, Г. Месси. Теория атомных столкновений (Мир, M., 1969) [N.F. Mott, H.S.W. Massey. Theory of Atomic Collisions (Oxford University Press, Oxford. 1965)]

[10] H.S.W. Massey, C.B.O. Mohr. Proc. R. Soc. Lond. A, 141, 434 (1933).

[11] J.F. Ziegler, J.P. Biersack, U. Littmark. The Stopping and Range of Ions in Solids (Pergamon, NY., 1985), v. 1.

[12] D.S. Meluzova, P.Yu. Babenko, A.P. Shergin, K. Nordlund, A.N. Zinoviev. NIMB, 460, 4 (2019). DOI: 10.1016/j.nimb.2019.03.037

[13] Y.R. Luo. Comprehensive Handbook of Chemical Bond Energies (CRC Press, Boca Raton, 2007)

[14] B. Darwent. Bond Dissociation Energies in Simple Molecules (NSRDS-NBS, 31, 1970)

[15] А.А. Радциг, Б.М. Смирнов. Справочник по атомной и молекулярной бизике (Атомиздат, Москва, 1980) [A.A. Radzig, B.M. Smirnov. Reference Data on Atoms, Molecules, and Ions, Springer Series in Chemical Physics (Springer, Berlin, 1985), v. 31.]

[16] Б.П. Никольский. Справочник химика (Химия, Л., 1966) [B.P. Nikolsky. Handbook of Chemist (Chemistry Publ. House, Leningrad, 1966)]

[17] NIST data base - https://webbook.nist.gov/chemistry/.

[18] J. Linchard, V. Nielsen, M. Scharff. Mat. Fys. Medd. Dan. Vid. Selsk., 36, 1 (1968).

[19] О.Б. Фирсов. ЖЭТФ, 34, 447 (1958) [О.В. Firsov. JЕTР, 7, 308 (1958).] 